\title{
Concurrent application of tretinoin (retinoic acid) partially protects against corticosteroid-induced epidermal atrophy
}

\author{
A.J.MCMICHAEL, C.E.M.GRIFFITHS, H.S.TALWAR, L.J.FINKEL, E.S.RAFAL, \\ T.A.HAMILTON AND J.J.VOORHEES \\ Dermatopharmacology Unit, Department of Dermatology. University of Michigan Medical Center. Ann Arbor, Michigan, U.S.A.
}

Accepted for publication 23 November 1995

Summary Cutaneous atrophy arising from prolonged use of potent topical corticosteroids has long been a concern. Thus, it would be advantageous to find an agent which protects against atrophy produced by corticosteroids but at the same time does not impair their anti-inflammatory effects. Recent work shows that topical all-trans retinoic acid (tretinoin) prevents skin atrophy in mice treated with topical corticosteroids, but such studies have not been performed in humans. We performed an 8-week clinical, histological and biochemical study to test the ability of tretinoin to enhance efficacy and inhibit atrophogenicity of topical corticosteroids, when used in the treatment of psoriasis. In each of 20 psoriasis patients, one plaque, and its perilesional skin, was treated once daily with betamethasone dipropionate and tretinoin $0 \cdot 1 \%$, and one plaque, and its perilesional skin, treated with once daily betamethasone dipropionate and tretinoin vehicle. There was no difference in the speed or degree of improvement in plaques treated with either the topical corticosteroid/tretinoin combination or with corticosteroid alone. Light microscopy revealed a $19 \%$ reduction in epidermal thickness. in corticosteroid-treated perilesional skin, as compared with a slight (1\%) increase in corticosteroid/ tretinoin-treated perilesional areas $(P=0 \cdot 067)$. Western blot analysis showed a $55 \%$ reduction in procollagen I aminopropeptide in perilesional skin treated with corticosteroid alone, as compared with a $45 \%$ reduction in corticosteroid/tretinoin-treated perilesional skin. These data indicate that the addition of tretinoin does not impair the efficacy of a topical corticosteroid, in the treatment of psoriasis, and partially ameliorates epidermal atrophy produced by the topical corticosteroid.

Topical corticosteroids are helpful for the treatment of inflammatory dermatoses but their prolonged use may be associated with cutaneous atrophy. Studies in mice have demonstrated that concurrently applied all-trans retinoic acid (tretinoin) may prevent cutaneous atrophy produced by topical corticosteroids. ${ }^{1}$ Lesnik et al. ${ }^{2}$ have demonstrated that tretinoin will prevent corticosteroidinduced skin atrophy in hairless mice, but without impairment of corticosteroid anti-inflammatory properties. Results of another study ${ }^{3}$ are also compatible with this observation, as tretinoin does not alter the vasoconstrictive properties of topical corticosteroids in normal human skin. Atopic dermatitis also responds well to a combination of tretinoin and a mid-potency topical steroid, without any increased irritation. ${ }^{4}$

Thus far, no controlled study in humans has assessed

Correspondence: Professor Christopher E.M.Griffiths, Section of Dermatology, Department of Medicine, University of Manchester School of Medicine, Clinical Sciences Building, Hope Hospital, Salford, M6 8HD, U.K. the ability of tretinoin to, either, protect against corticosteroid-induced cutaneous atrophy or enhance the anti-inflammatory properties of corticosteroids. To address these questions, we investigated the ability of topical tretinoin to prevent atrophy of surrounding psoriatic skin during treatment with a topical corticosteroid, and to enhance the efficacy of a midpotency topical corticosteroid, during the treatment of psoriatic plaques.

\section{Methods}

Patients

Twenty, healthy psoriatic patients (17 men, three women; age range $25-70$, mean age 46 years), each with at least three stable psoriatic plaques of similar severity and size, were entered into an 8-week, doubleblind vehicle-controlled study. No topical or oral treatments, including ultraviolet radiation, other than bland 
emollients, were allowed within the 4 weeks preceding the study, or during the study itself. Pregnant and nursing women were excluded. All patients gave written informed consent, and the protocol was approved by the institutional review board of the University of Michigan Medical Center.

Three, clinically similar, psoriatic plaques were chosen, on each patient. One plaque remained untreated and served as a control. The other two plaques were treated each morning with a mid-potency topical corticosteroid, $0 \cdot 05 \%$ betamethasone dipropionate cream (BD; $0 \cdot 05 \%$ Diprosone. Schering Corporation, Kenilworth, New Jersey, U.S.A.) and were randomized to evening treatment with either $0 \cdot 1 \%$ tretinoin cream (Retin- $A^{\text {TM }}$, Ortho Pharmaceutical Corporation, Raritan, New Jersey, U.S.A.) or colourmatched vehicle cream (Ortho). Each study plaque was a minimum of $5 \mathrm{~cm}$ in diameter, to ensure sufficient biopsy area, and all treatments were applied to the active plaque and to $5 \mathrm{~cm}$ of uninvolved perilesional skin. No study drugs were applied on the evening before, or on the morning of, scheduled clinic visits. All patients received a mild emollient to be used, when needed, for skin irritation or dryness. As all dispensed tubes were identical in appearance, both investigators and patients were unaware of the treatment group to which each plaque was assigned. The sample sizes were chosen to provide a statistical power of approximately $0 \cdot 80$ to detect a mean difference of $15 \mu \mathrm{m}$ in epidermal thickness, at the end of therapy, between $\mathrm{BD} /$ tretinoin and $\mathrm{BD} /$ vehicle at a type I error rate of $0 \cdot 05$, for a twosided paired comparison.

\section{Clinical evaluations}

Clinical evaluations were performed at baseline, and at weeks $1,2,4,6$ and 8 of therapy. One investigator (AJM) evaluated all patients at all visits. At the baseline visit only, an overall evaluation of the severity of psoriasis for all three plaques was performed, using a five-point scale where 1 indicates no psoriasis; 2, mild; 3 , moderate; 4 , severe and 5 , very severe. At both baseline and follow-up visits, scaling, erythema and plaque elevation were each assessed for each plaque on a nine-point, $0-8$ scale. At follow-up visits only, a global evaluation, compared with baseline, for all plaques, was performed on a seven-point scale with 1 indicating markedly worse; 2 , worse; 3 , slightly worse; 4 , no change; 5 , slightly improved; 6 , improved; and 7 . markedly improved. Also, skin atrophy of all three plaques, and their corresponding perilesional skin, was evaluated on a six-point scale with 1 indicating none; 2 , trace; 3 , mild; 4 , moderate; 5 , marked; and 6 , severe.

\section{Side-effects}

At each visit, cutaneous side-effects at all treatment sites, and their corresponding perilesional skin, were evaluated by measuring erythema, peeling, itching, burning/stinging, depression, striae, wrinkling and telangiectasia, using the aforementioned six-point scale. For cutaneous effects, only increases of two or more units, over baseline, at two or more visits, were evaluated statistically.

\section{Light microscopy}

Two, $4 \mathrm{~mm}$ punch biopsies, one from the plaque and one from perilesional skin of each treated plaque, were obtained at baseline and after 8 weeks of treatment. The biopsies were immediately bisected, and one-half placed in $10 \%$ neutral-buffered formalin, embedded in paraffin. sectioned, and stained with haematoxylin and eosin. The other half of the specimen was either placed in optimal cutting temperature medium (OCT, Miles Laboratories, Elkhart, Indiana, U.S.A.) and snap frozen in liquid nitrogen, or directly frozen in liquid nitrogen. and stored at $-70^{\circ} \mathrm{C}$ until processed for Western blot analysis. The investigator who analysed the histological specimens (LJF) was not aware of either the patient's treatment or the timing of the biopsy. Stratum corneum compaction, granular cell layer thickness, spongiosis and dermal inflammation were assessed using a semiquantitative scale ranging from 0 to 4 , where 0 was no evidence of parameter, and 4 was maximum expression. Epidermal thickness in $\mu \mathrm{m}$ was measured from the top of the granular layer to the epidermal basement membrane, in five inter rete-ridge spaces per specimen.

\section{Western blot analysis}

Procollagen was extracted from skin biopsies, as described, ${ }^{5,6}$ and supernatants prepared for Western blot analysis of type I procollagen aminopropeptide (pNcol I). Incubations were performed with affinity purified anti-pNcol I polyclonal antibody (a generous gift of Drs J. and L.Ristelli, University of Oulu, Finland) overnight at $4{ }^{\circ} \mathrm{C}$, and $\mathrm{pNcol} \mathrm{I}$ identified by comigration with authentic standards (a generous gift of Prof. M.Lapiere, University of Liege, Belgium). Radioactivity in the bands was measured by Phosphorimager 
(Molecular Dynamics), and results expressed as counts per minute/unit protein.

\section{Statistical analysis}

Comparisons of the change from baseline in clinical and histological parameters, between sites treated with corticosteroid alone, vs. those treated with corticosteroid plus tretinoin, were made with the paired $t$-test. The nonparametric Wilcoxon signed-rank test was used to compare changes in pNcol I levels, as determined by Western blot analysis due to the non-normal distribution of the data. All $P$ values were two-tailed. Summary statistics are expressed as means \pm standard error of the mean (SEM). The data were analysed with the use of the Michigan Interactive Data Analysis System (MIDAS), a statistical software package developed by the Centre for Statistical Consultant and Research, at the University of Michigan.

\section{Results}

Seventeen of 20 patients completed the 8-week study, although only 16 of them had the final 8-week biopsy performed. Two patients dropped out, one after week 4 , and one after week 6 , because of personal responsibilities. One patient discontinued the study at week 1 , secondary to a protocol violation of applying topical study creams to the control site. One patient refused biopsy upon completion of the study. Therefore, clinical data are presented for 17 patients and histological data for 16 patients.

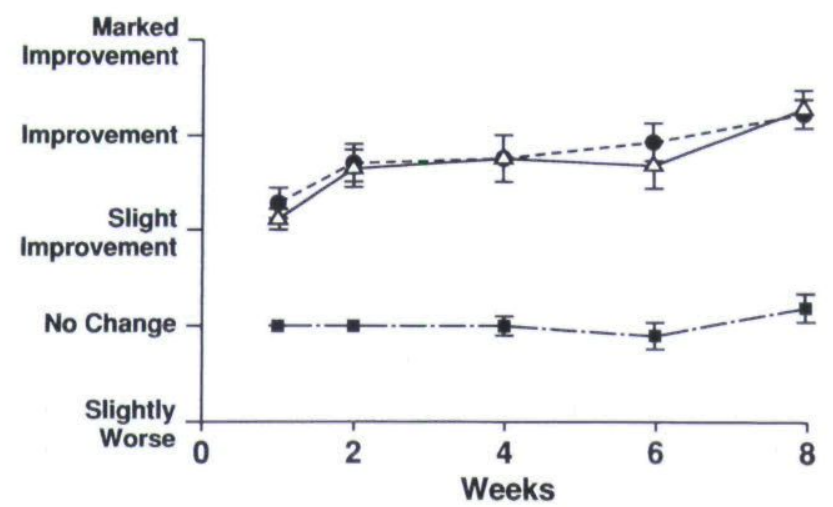

Figure 1. Global response to treatment of psoriasis plaques with betamethasone dipropionate (BD) vehicle (broken lines), BD/tretinoin (solid lines) or no treatment (alternate, long-short dashed lines). Circles, squares and triangles with error bars indicate means \pm standard error (SE), $n=17$. No statistically significant difference was noted in the rate or overall improvement of $\mathrm{BD} /$ vehicle treated plaques vs. the $\mathrm{BD} /$ tretinoin treated plaques.

\section{Clinical findings}

Assessment of the clinical global response revealed that all patients had at least slight improvement in their psoriasis, at both treated sites, after 8 weeks of treatment. Sites treated with $\mathrm{BD} /$ tretinoin showed a marked improvement in nine (53\%) patients, an improvement in four $(24 \%)$ and a slight improvement in four. Similarly, sites treated with $\mathrm{BD} /$ vehicle demonstrated a marked improvement in nine $(53 \%)$ patients, an improvement in three $(18 \%)$, and a slight improvement in five $(29 \%)$. Control, untreated sites, showed a slight improvement in five $(29 \%)$ patients, no change in $10(59 \%)$, and a worsening in two $(12 \%)$. The speed of improvement was no different between the two treatment sites. Thus, the two treatments were equally efficacious and the addition of tretinoin conferred no added advantage in efficacy to BD for psoriasis treatment (Fig. 1). Furthermore, at completion of the study (week 8), no clinical atrophy was noted in any perilesional area.

\section{Side-effects}

Cutaneous reactions were characterized principally by erythema and scaling. Erythema and/or scaling of at least moderate severity occurred in six of $19(32 \%)$ patients, in the tretinoin-treated plaques, and in one of $19(5 \%)$ patients, in the vehicle-treated plaques, during the study.

\section{Histological findings}

In perilesional skin treated with $\mathrm{BD} /$ vehicle, the epi-

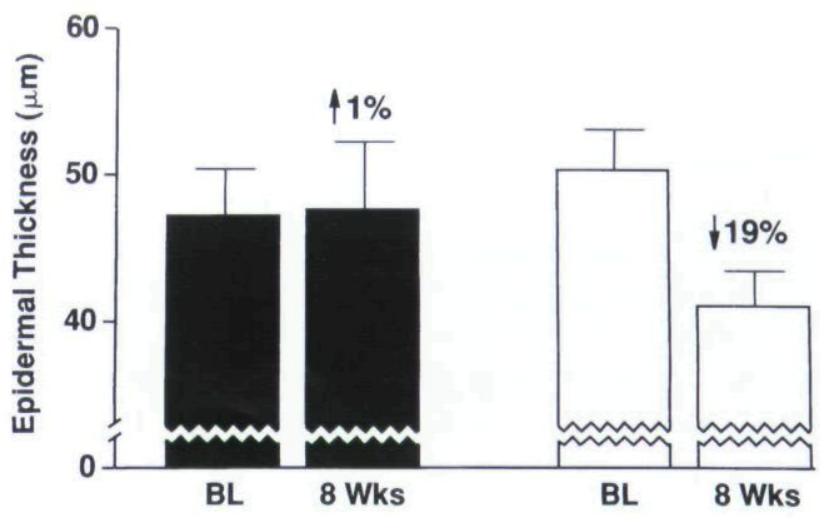

Figure 2. Overall change from baseline (BL) in epidermal thickness of perilesional skin treated with betamethasone dipropionate (BD)/tretinoin or $\mathrm{BD} /$ vehicle for 8 weeks. $\mathrm{BD} /$ tretinoin combination (solid bars) protected against the marked decrease in epidermal thickness effected by the $\mathrm{BD} /$ vehicle combination (open bars) $(P=0.067)$. Bars are means \pm SE, $n=16$. 
Figure 3. Effects of 8 weeks treatment with betamethasone dipropionate (BD)/vehicle or $\mathrm{BD} /$ tretinoin on epidermal thickness. Baseline, pretreatment perilesional skin biopsies (week 0) from one patient show normal epidermal histology and thickness. Eight weeks treatment with $\mathrm{BD} /$ vehicle produces appreciable thinning of the epidermis (week 8), in contrast to 8 weeks treatment with $\mathrm{BD} /$ tretinoin which protects against BD-induced epidermal thinning (week 8). (Haematoxylin and eosin; $\times 100$.)
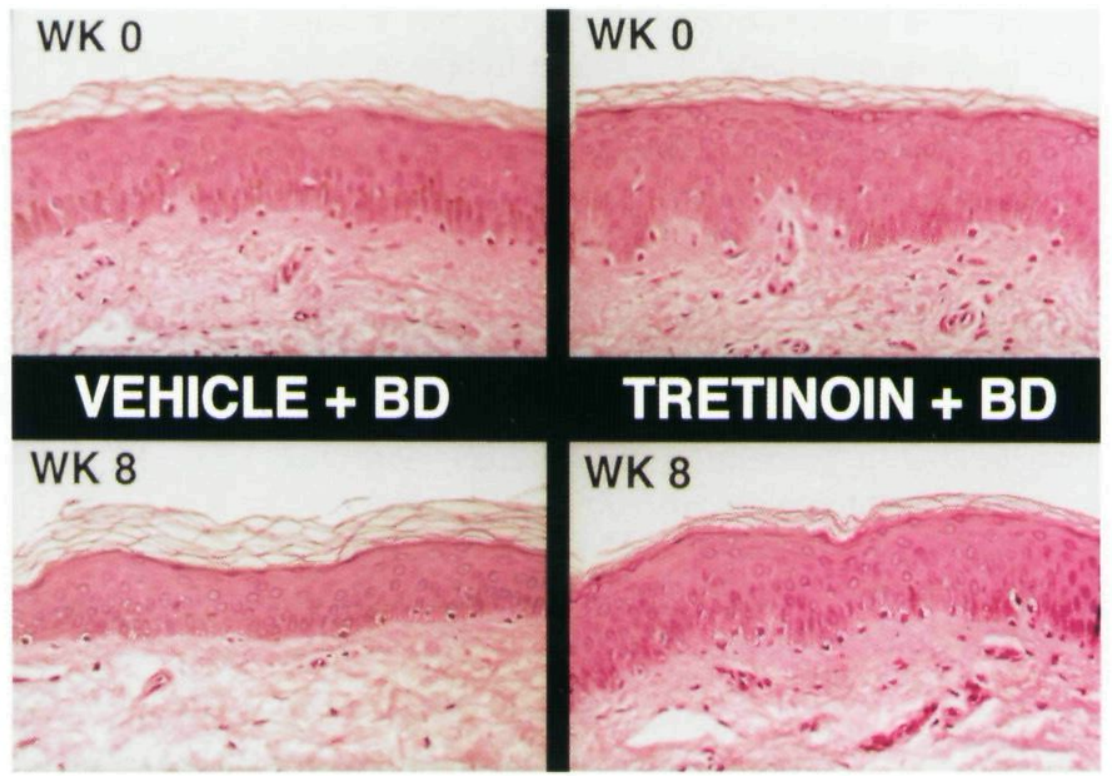

dermal thickness decreased (19\%) from $50 \pm 3$ to $41 \pm 2 \mu \mathrm{m}$, as compared with a slight, $1 \%$, increase in epidermal thickness from $47 \pm 3$ to $48 \pm 3 \mu \mathrm{m}$, in the $\mathrm{BD} /$ tretinoin sites $(P=0.067$, Figs 2 and 3$)$. Thus, tretinoin partially abrogates epidermal atrophy produced by topical corticosteroid use.

Stratum corneum was compacted significantly from $0 \cdot 2 \pm 0 \cdot 2$ units, at baseline, to $1 \cdot 5 \pm 0 \cdot 4$ units, at week 8 , in the $\mathrm{BD} /$ tretinoin group, as compared with no compaction in the $\mathrm{BD} /$ vehicle group, $0 \cdot 7 \pm 0 \cdot 3$ units at baseline, to $0 \cdot 2 \pm 0 \cdot 1$ units, at the end of treatment $(P<0 \cdot 001)$. Also, granular layer thickness increased significantly in the $\mathrm{BD} /$ tretinoin group, from $0 \cdot 7 \pm 0 \cdot 1$ to $1 \cdot 3 \pm 0 \cdot 2$, as compared with $0 \cdot 8 \pm 0 \cdot 1$ to $0 \cdot 5 \pm 0 \cdot 03$, in the $\mathrm{BD} /$ vehicle group $(P=0 \cdot 001)$

\section{Western blot analysis}

Owing to three specimens not being snap frozen, only 13 biopsy samples were available for analysis by Western blotting. In perilesional skin treated for 8 weeks with $\mathrm{BD} /$ vehicle, there was a $55 \%$ reduction in pNcol I, from $1211 \pm 365$ at baseline to $458 \pm 161$ at 8 weeks. In $\mathrm{BD} /$ tretinoin treated skin, there was a lesser, $45 \%$, reduction in pNcol I, from $1178 \pm 338$ at baseline to $650 \pm 208$ at 8 weeks $(P=0 \cdot 07)$.

\section{Discussion}

The combination of topical corticosteroids and tretinoin for the treatment of psoriasis is not a new idea. As early as 1973, it was demonstrated that fluorinated corticosteroids combined with tretinoin-cleared psoriatic plaques, perhaps with enhanced clinical therapeutic effect. ${ }^{7}$ Kaidbey et al. ${ }^{8}$ showed that combined therapy with $0 \cdot 1 \%$ tretinoin and potent topical corticosteroid was efficacious in psoriasis, but the issue of skin atrophy was not addressed. We observed no difference in the speed of resolution nor in the degree of improvement of psoriatic plaques treated with corticosteroid and vehicle, as compared with plaques treated with corticosteroid and tretinoin for 8 weeks. We did not observe that the addition of $0 \cdot 1 \%$ tretinoin increased corticosteroid efficacy, but this may be because efficacy of the corticosteroid that we used (BD) may already be very close to, or at the peak of, the dose-response curve. Thus, it still remains that efficacy of a mild potency topical corticosteroid could be enhanced by the addition of tretinoin.

Although we could not demonstrate clinical atrophy in skin treated with corticosteroid alone, there was both histological and biochemical evidence that atrophy had occurred. Furthermore, the addition of tretinoin to the corticosteroid protected, to some extent, against epidermal atrophy. The 19\% decrease in epidermal thickness during corticosteroid treatment was similar to that observed by Lesnik et al. ${ }^{2}$ in the mouse ear model, where potent topical corticosteroids thinned the epidermis to two cell layers. In our study, the significant compaction of stratum corneum and thickening of granular layer, with the addition of tretinoin, are markers of retinoid activity and per se may not be evidence of a protective effect of tretinoin. 
In addition, we investigated collagen precursor content in the dermal matrix. It is known from studies in rats, ${ }^{9}$ that triamcinolone diacetate decreases synthesis of type I procollagen, in addition to other collagens. Furthermore, it has recently been demonstrated that topical tretinoin can restore synthesis of collagen I in photoaged human skin. ${ }^{10}$ and collagen III in photoaged mouse skin. ${ }^{11} \mathrm{pNcol}$ I was reduced to a lesser, although non-significant, extent in the corticosteroid/tretinointreated sites as compared with corticosteroid alone, implying that topical retinoids may partially abrogate corticosteroid-induced decreases in collagen synthesis. Further study is required to prove that it is indeed the synthesis, and not rate of breakdown, of collagen that is affected by topical tretinoin.

\section{Acknowledgments}

Supported in part by the R.W.Johnson, Pharmaceutical Research Institute, Raritan, New Jersey and the Babcock Dermatologic Endowment, Ann Arbor, Michigan.

\section{References}

1 Mezick JA, Gendimenico GJ. Kligman LH et al. Prevention and reversal of glucocorticoid-induced skin atrophy by retinoids in mouse skin. Dermatologica 1991: 181: 349-50.
2 Lesnik RH, Mezick JA, Capetola RJ, Kligman LH. Topical all-trans retinoic acid prevents corticosteroid-induced skin atrophy without abrogating the anti-inflammatory effect. I Am Acad Dermatol 1989: 21: 186-90.

3 Schmied C, Saurat JH. Topical retinoic acid does not alter the vasoconstrictive properties of topical corticosteroids in humans. Dermatologica 1991: 182: 107-11.

4 Schmied C, Piletta PA. Saurat JH. Treatment of eczema with a mixture of triamcinolone acetonide and retinoic acid: a doubleblind study. Dermatology 1993; 187: 263-7.

5 Smith BD, McKenney KH, Lustberg TJ. Characterization of collagen precursors found in rat skin and rat bone. Biochemistry 1977: 16: 2980-5.

6 Talwar HS, Griffiths CEM, Fisher GJ, Voorhees JJ. Reduced type I and type III procollagens in photodamaged adult human skin. J Invest Dermatol 1995: 105: 285-90.

7 Orfanos CE, Schmidt HW, Mahre G et al. Retinoic acid in psoriasis: its value for topical therapy with and without corticosteroids. $\mathrm{Br}]$ Dermatol 1973: 88: 167-82.

8 Kaidbey KH, Petrozzi JW, Kligman AM. Treatment of psoriasis with topically applied tretinoin and steroid ointment. Arch Dermatol 1975: 111: 1001-3.

9 Shull S, Cutroneo KR. Glucocorticoids coordinately regulate procollagens type I and type II synthesis. J Biol Chem 1983; 258: 3364-9.

10 Griffiths CEM, Russman AN, Majmudar G et al. Restoration of collagen formation in photodamaged skin by tretinoin (retinoic acid). N Engl J Med 1993; 329: 530-5.

11 Schwartz E, Cruikshank FA, Mezick JA. Kligman LH. Topical alltrans retinoic acid stimulates collagen synthesis in vivo. I Invest Dermatol 1991: 96: 975-8. 
This document is a scanned copy of a printed document. No warranty is given about the accuracy of the copy. Users should refer to the original published version of the material. 